\title{
La actividad física regular disminuye la mortalidad global en mujeres postmenopáusicas.
}

Physical activity and mortality in Postmenopausal Women. Kushi L, Fee R, Folsom A, et al. JAMA 1997; 277:1287-1292

\section{Objetivo}

Evaluar la asociación entre la actividad física regular y la mortalidad global en mujeres postmenopáusicas.

\section{Diseño}

Estudio poblacional de cohortes con 7 años de seguimiento.

\section{Lugar}

Estado de Iowa, EE.UU.

\section{Participantes}

Mujeres entre 55 y 69 años de edad seleccionadas al azar de la población general que respondieron una encuesta de salud $(n=41.836)$.

\section{Evaluación de factores de riesgo}

El cuestionario incluía preguntas sobre tabaquismo, dieta, alcohol, antecedentes médicos, paridad, historia familiar, peso y altura. Las participantes fueron asignadas a una de tres categorías de acuerdo a sus respuestas sobre intensidad y frecuencia de la actividad física que desarrollaban: leve ( actividad moderada o intensa pocas veces al mes, $n=18.940$ ), moderada (actividad intensa 1/semana o moderada 1 a 4 / semana, $\mathrm{n}=10.987$ ) o intensa ( moderada actividad $>4$ /semana, $\mathrm{n}=9919$ )

\section{Medición de resultados principales \\ Las 2284 muertes ocurridas hasta diciembre 1992 fueron identificadas a través del registro estatal y na- cional de defunciones. La causa de muerte estaba co-}

dificada según el código internacional de enfermedades (CIE-9). Hubo 1101 casos de cáncer, 739 de causa cardiovascular, 150 respiratorias y 237 por otras causas.

\section{Resultados principales}

La intensidad y frecuencia de actividad física aún dentro de la misma categoría mostró una relación inversa progresiva con la mortalidad.

\begin{tabular}{|l|l|l|l|l|}
\hline & & $\begin{array}{l}\text { Muertes } \\
\text { (seguimiento) }\end{array}$ & $\begin{array}{l}\text { Personas/año } \\
{[\text { [C 95\% }]^{*}}\end{array}$ & RR ajustado \\
\hline $\begin{array}{l}\text { Actividad } \\
\text { física regular }\end{array}$ & no & 1518 & 157.379 & 1.00 \\
\hline si & 742 & 111.811 & $0,78[0,71 \cdot 0,86]$ \\
\hline Indice de & bajo & 1309 & 126.545 & 1.00 \\
act. física & moderado & 519 & 74.170 & $0,77[0,69-0,86]$ \\
& intenso & 415 & 67.738 & $0,68[0,60-0,77]$ \\
\hline
\end{tabular}

* Ajustado por edad basal, menarca, menopausia, paridad, nivel de educación estado civil ,consumo de alcohol, tabaquismo uso de estrógenos, obesidad, historia de cáncer, HTA, diabetes.

\section{Conclusiones}

La actividad física regular se asocia con una reducción de la mortalidad global en mujeres postmenopáusicas. Aún la actividad moderada una vez por semana reduce el riesgo de muerte comparando con un estilo de vida sedentario. tividad física sino también a las diferencias entre las distintas poblaciones estudiadas. Los estudios epidemiológicos observacionales son frecuentemente la única fuente de la cual se obtiene información sobre distintos factores de riesgo y el desarrollo de enfermedades o muerte. Bradford Hill definió en 1971 los principios que refuerzan la idea de una asociación causal entre la exposición y el riesgo o beneficio estimado en un estudio; de estos los más importantes son: temporalidad (la exposición debe preceder al evento); fuerza de asociación (el aumento o reducción del riesgo debe ser clínicamente significativo); consistencia (demostrar la misma tendencia que otros estudios similares); efecto dosis - respuesta y plausibilidad biológica. Ambos estudios comparten detalles metodológicos que fortalecen sus conclusiones: 1) Son cohortes prospectivas y poblacionales numerosas (participantes elegidas a través de encuestas a la población general seguidas en el tiempo); 2) La reducción del riesgo de 20 a $60 \%$ asociada a la actividad física es muy importante para un fenómeno multicausal; 3) La asociación es consistente con otros estudios; 4) Presentan gradiente dosis - respuesta (a mayor actividad menor riesgo) y 5) la asociación esta sustentada por fenómenos que tienen explicación fisiopatológica, es decir, son plausibles biológicamente. En resumen, la actividad física es claramente beneficiosa y estos resultados no hacen más que confirmar y reforzar la necesidad de insistir con el consejo sobre ejercicio regular a toda mujer independientemente de su edad.

\section{Dr Fernando Rubinstein \\ Unidad de Medicina Familiar y Preventiva Hospital Italiano de Buenos Aires}

\section{Referencias}

Sherman S, D'Agostino R, Cobb J, Kannel W. Physical activity and mortality in women in the Framingham Heart Study. Am Heart J 1994; 128:879-884

Sherman S, D'Agostino R, Cobb J, Kannel W. Does exercise reduce mortality rates in the elderly? Am Heart J 1993; 83:1443:1450.

Leon A, Connet J, for the MRFIT research group. Physical activity and 10.5 year mortality in the Multiple Risk factor Intervention Trial. Int J Epidemiol. 1991;20:690-697.

Hill B. Principles of medical statistics. New York: Oxford University press, 1971. 